\title{
A goal programming method for deriving fuzzy priorities of criteria from inconsistent fuzzy comparison matrices
}

\author{
Mohammad Izadikhah*
}

Department of Mathematics, Islamic Azad University, Arak Branch, Arak, Iran

\begin{tabular}{l}
\hline A R T I C L E I N F O \\
\hline Article history: \\
Received July 20, 2011 \\
Received in Revised form \\
September, 22, 2011 \\
Accepted 4 October 2011 \\
Available online \\
7 October 2011 \\
\hline Keywords: \\
Triangular fuzzy number \\
Fuzzy pair-wise comparison \\
matrix \\
Goal programming \\
Ranking function
\end{tabular}
A B S T R A C T

Decision making problem is the process of finding the best option from all of the feasible alternatives. One of the most important concepts in decision making process is to identify the weights of criteria. In real-world situation, because of incomplete or non-obtainable information, the data (attributes) are often not deterministic and can be treated in forms of fuzzy numbers. This paper investigates a method for deriving the weights of criteria from the pairwise comparison matrix with fuzzy elements. Finding the weights of criteria has been one of the most important issues in the field of decision-making and the present method uses goal programming to solve the resulted model. In addition, using a ranking function we convert each obtained fuzzy weight to a crisp one, which makes it possible to compare the criteria. The proposed model of this paper is supported by several examples and a case study.

(C) 2012 Growing Science Ltd. All rights reserved.

\section{Introduction}

In evaluating $n$ competing alternatives $A_{1}, \ldots, A_{n}$ under a given criterion, it is natural to usethe framework of pairwise comparisons represented by an $\times n$ square matrix from which a set of preference values for the alternatives is derived. Because of ease of understanding and application, pairwise comparisons play an important role in assessing the priority weights of decision criteria. Geoffrion's gradient search method (1972), Haimes' surrogate worth tradeoff method (1980), ZiontsWallenius' method (1976), Saaty's analytic hierarchy process (1980), Cogger and Yu's eigenvector method (1985), Takeda, Cogger and Yu's GEM (1987), and the logarithmic least square method (Crawford and Williams (1985)) are just some methods which are primarily based on pairwise comparisons. Pairwise comparison matrices are often used in Multi-attribute Decision Making for weighting the attributes or for the evaluation of the alternatives with respect to criteria.

Determining criteria weights is a central problem in multi-criteria decision making (MCDM). Weights are used to express the relative importance of criteria in MCDM. The determination of weights are required when applying MCDM methods such as goal programming, the analytic hierarchy process (AHP), and the weighted score method. In practice, it is difficult for decision maker

* Corresponding author. Tel: + 988613663041

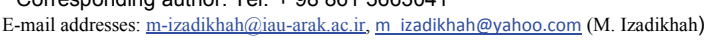


to supply relative numerical weights of different decision criteria. Quite often, decision makers are much more comfortable in simply assigning ordinal ranks to the different criteria under consideration. In such cases, relative criteria weights can be derived from criteria ranks supplied by decision makers.

The classical pair-wise comparison matrix requires the decision maker (DM) to express his/her preferences in the form of a precise ratio matrix encoding a valued preference relation. However it can often be difficult for the DM to express exact estimates of the ratios of importance and therefore express his/her estimates as fuzzy numbers.

The theory of fuzzy numbers is based on the theory of fuzzy sets, which Zadeh introduced in 1965. First, Bellman and Zadeh (1970) incorporated the concept of fuzzy numbers into decision analysis. The methodology presented in this paper is useful in assisting decision makers to determine criteria fuzzy weights from criteria, and it is helpful in alternative selection when these fuzzy weights are used with one of the techniques of MCDM.

To deriving the weights of criteria from this fuzzy pair-wise comparison matrix is an important problem. Islam et al. (1997) and Wang (2006) developed a lexicographic goal programming to generate weights from inconsistent pair-wise interval comparison matrices. Many methods for estimating the preference values from the pairwise comparison matrix have been proposed and their effectiveness comparatively evaluated. Some of the proposed estimating methods presume intervalscaled preference values (Barzilai, (1997) and Salo, (1997)).

In this paper we apply the goal programming method to derive fuzzy weights of criteria. Goal programming was originally proposed by Charnes and Cooper (1961), and is an important technique for DMs to consider simultaneously several objectives in finding a set of acceptable solution. Also in order to compare the criteria we use the ranking function proposed by Asady and Zendehnam (2007). Technique for order performance by similarity to ideal solution (TOPSIS), one of known classical MCDM method, was first developed by Chen and Hwang (1992), with reference to Hwang and Yoon (1987), for solving a MCDM problem. TOPSIS, known as one of the most classical MCDM methods, is based on the idea, that the chosen alternative should have the shortest distance from the positive ideal solution and on the other side the farthest distance of the negative ideal solution. Recently, in some research, the TOPSIS method is considered for extension. For example, Chen (2000) extended the concept of TOPSIS to develop a methodology for solving multi-person multi-criteria decisionmaking problems in a fuzzy environment. Abo-Sinna et al. (2005) extended the TOPSIS method to solve multi-objective nonlinear programming problems. Also, Jahanshahloo et al. (2006,a,b) and Izadikhah (2009) extended the TOPSIS method for decision making problems with interval and fuzzy data.

This paper also extends the concept of TOPSIS to develop a methodology for solving multi-criteria decision-making problems with fuzzy data. For this task, we use the fuzzy weights obtained by the proposed method.

The structure of the rest of this paper is following: The following section provides some required preliminaries. The third section of the paper gives a goal programming approach for deriving weights of criteria. Two examples and a case study are presented in section 4. The paper ends with conclusion.

\section{Preliminaries}

In this section we review some basic definitions about fuzzy numbers, fuzzy pair-wise comparison matrix and goal programming method. 


\subsection{Fuzzy numbers}

Fuzzy numbers are one way to describe the vagueness and lack of precision of data. The theory of fuzzy numbers is based on the theory of fuzzy sets, which Zadeh introduced in 1965.

\subsubsection{Some basic definitions of fuzzy numbers}

Definition 1. A fuzzy number is a fuzzy set like $\mu_{A}: R \rightarrow I=[0,1]$ which satisfies:

- $\mu_{A}$ is continuous,

- $\mu_{A}(x)=0$ outside some interval [a,d],

- There are real numbers $\mathrm{b}, \mathrm{c}$ such that $a \leq b \leq c \leq d$

and

1. $\mu_{A}(x)$ is increasing on $[\mathrm{a}, \mathrm{b}]$,

2. $\mu_{A}(x)$ is decreasing on $[\mathrm{c}, \mathrm{d}]$,

3. $\mu_{A}(x)=1, b \leq x \leq c$.

We denote the set of all fuzzy numbers by $F(R)$. Parametric form of fuzzy numbers is defined in Asady and Zendehnam (2007) as follows:

Definition 2. A fuzzy number $\tilde{A}$ in parametric form is a pair $(\underline{A}(r), \bar{A}(r))$ of functions $\underline{A}(r), \bar{A}(r)$, $0 \leq r \leq 1$, which satisfies the following requirements:

1. $\underline{A}(r)$ is a bounded increasing continuous function,

2. $\bar{A}(r)$ is a bounded decreasing continuous function,

3. $A(r) \leq \bar{A}(r), \quad 0 \leq r \leq 1$.

A crisp number $\lambda$ is simply represented by $\underline{A}(r)=\bar{A}(r)=\lambda, \quad 0 \leq r \leq 1$.

Definition 3. ( $\alpha$-level set or $\alpha$-cut). The $\alpha$-cut of a fuzzy set $\tilde{A}$ is a crisp subset of $X$ and is denoted by:

$[\tilde{A}]_{\alpha}=\left\{x \mid \mu_{\tilde{A}}(x) \geq \alpha\right\}$,

where $\mu_{\tilde{A}}(x)$ is the membership function of $\tilde{A}$ and $\alpha \in[0,1]$.

Definition 4. A triangular fuzzy number is denoted as $\tilde{A}=(a, b, c)$, see Fig. 1 .

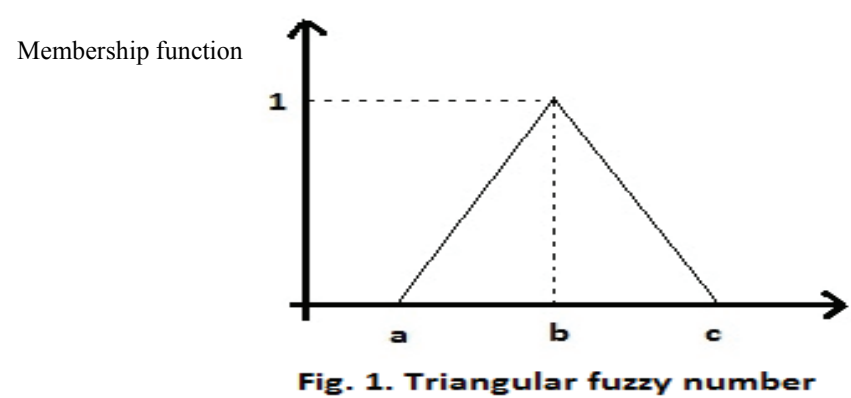

The membership function of a triangular fuzzy number is express as 
$\mu_{A}(x)= \begin{cases}\frac{x-a}{b-a}, & a \leq x \leq b \\ \frac{c-x}{c-b}, & b \leq x \leq c \\ 0, & \text { Otherwise }\end{cases}$

Definition 5. A fuzzy number $\tilde{A}=(a, b, c)$ is set to be non-negative fuzzy number, if and only if $a \geq 0$.

Corollary 1.The parametric form of triangular fuzzy number $\tilde{A}=(a, b, c)$ is obtained as:

$\tilde{A}=(\underline{A}(r), \bar{A}(r))=\left\{\begin{array}{l}\underline{A}(r)=a+(b-a) r \\ \bar{A}(r)=c-(c-b) r\end{array}\right.$

Definition 6. (Multiplication of triangular fuzzy numbers) Suppose that we have two triangular fuzzy numbers $\tilde{A}$ and $\tilde{B}$ such that $\tilde{A}=\left(a_{1}, a_{2}, a_{3}\right)$ and $\tilde{B}=\left(b_{1}, b_{2}, b_{3}\right)$, then, the multiplication of the fuzzy numbers $\tilde{A}$ and $\tilde{B}$ isdefined as follows:

$\tilde{A} \cdot \tilde{B}=\left(a_{1} b_{1}, a_{2} b_{2}, a_{3} b_{3}\right)$

Definition 7. Let $\tilde{A}=\left(a_{1}, a_{2}, a_{3}\right)$ and $\tilde{B}=\left(b_{1}, b_{2}, b_{3}\right)$ be two triangular fuzzy numbers, then the distance between them using vertex method is defined as

$d(\tilde{A}, \tilde{B})=\sqrt{\frac{1}{3}\left(\left(a_{1}-b_{1}\right)^{2}+\left(a_{2}-b_{2}\right)^{2}+\left(a_{3}-b_{3}\right)^{2}\right)}$

\subsubsection{Comparison between two fuzzy numbers}

In this subsection, in order to compare two fuzzy numbers, we use the concept of ranking function. A ranking function is a function $g: \mathrm{F}(R) \rightarrow R$, which maps each fuzzy number into the real line, where a natural order exists. Asady and Zendehnam proposed a defuzzification using minimizer of the distance between two the fuzzy number. They introduced distance minimization of a fuzzy number $\tilde{A}$ that denoted by $\mathrm{M}(\tilde{A})$ which was defined as follows:

$M(\tilde{A})=\frac{1}{2} \int_{0}^{1}(\bar{A}(r)+\underline{A}(r)) d r$

This ranking function have the following properties:

Property 1. If $\tilde{A}$ and $\tilde{B}$ be two fuzzy numbers then:

- $\quad \mathrm{M}(\tilde{A})>\mathrm{M}(\tilde{B})$ iff $\tilde{A} \succ \tilde{B}$,

- $\mathrm{M}(\tilde{A})<\mathrm{M}(\tilde{B})$ iff $\tilde{A} \prec \tilde{B}$,

- $\mathrm{M}(\tilde{A})=\mathrm{M}(\tilde{B})$ iff $\tilde{A} \approx \tilde{B}$,

Property 2. If $\tilde{A}$ and $\tilde{B}$ be two fuzzy numbers then:

$\mathrm{M}(\tilde{A} \oplus \tilde{B})=\mathrm{M}(\tilde{A})+\mathrm{M}(\tilde{B})$ 
Property 3.If $\tilde{A}=(a, b, c)$ be a triangular fuzzy number, then we have:

$M(\tilde{A})=\frac{1}{4}\{a+2 b+c\}$

\subsection{Fuzzy pair-wise comparison matrix}

Suppose the decision maker provides fuzzy judgments instead of precise judgments for a pair-wise comparison. Without loss of generality we assume that we deal with pair-wise comparison matrix with triangular fuzzy numbers being the elements of the matrix. We consider a pair- wise comparison matrix where all its elements are triangular fuzzy numbers as follows

$$
\tilde{A}=\left[\begin{array}{ccc}
\left(a_{11}^{L}, a_{11}^{M}, a_{11}^{U}\right) & \ldots & \left(a_{1 n}^{L}, a_{1 n}^{M}, a_{1 n}^{U}\right) \\
\vdots & \ddots & \vdots \\
\left(a_{n 1}^{L}, a_{n 1}^{M}, a_{n n}^{U}\right) & \ldots & \left(a_{n n}^{L}, a_{n n}^{M}, a_{n n}^{U}\right)
\end{array}\right]
$$

Where $\tilde{a}_{i j}=\left(a_{i j}^{L}, a_{i j}^{M}, a_{i j}^{U}\right)$ is a triangular fuzzy number, see Chen et al. (1992). We say that $\tilde{A}$ is reciprocal, if the following condition is satisfied (Ramik \& Korviny, 2010):

$\tilde{a}_{i j}=\left(a_{i j}^{L}, a_{i j}^{M}, a_{i j}^{U}\right)$

implies $\tilde{a}_{j i}=\left(\frac{1}{a_{i j}^{U}}, \frac{1}{a_{i j}^{M}}, \frac{1}{a_{i j}^{L}}\right)$ for all $i, j=1, \ldots, n$.

\subsection{Goal programming}

Consider the following problem:

$\max \left\{f_{1}(x), \ldots, f_{k}(x)\right\}$

subject to $x \in X$

where $f_{1}, \ldots, f_{k}$ are objective functions and $X$ is nonempty feasible region. Model (10) is called multiple objective programming. Goal programming is now an important area of multiple criteria optimization. The idea of goal programming is to establish a goal level of achievement for each criterion. In goal programming method requires the decision maker to set goals for each objective that he/she wishes to obtain. A preferred solution is then defined as the one, which minimizes the deviations from the set goals. Then GP can be formulated as the following achievement function.

$\min \sum_{i=1}^{k}\left(d_{i}^{+}+d_{i}^{-}\right)$

subject to

$$
\begin{array}{ll}
f_{i}(x)+d_{i}^{+}-d_{i}^{-}=b_{i}, & i=1, \ldots, k, \\
x \in X, & \\
d_{i}^{+} d_{i}^{-}=0, & i=1, \ldots, k, \\
d_{i}^{+}, d_{i}^{-} \geq 0, & i=1, \ldots, k,
\end{array}
$$


The DMs for their goals set some acceptable aspiration levels, $b_{i}(i=1, \ldots, k)$, for these goals, and try to achieve a set of goals as closely as possible. The purpose of GP is to minimize the deviations between the achievement of goals, $f_{i}(x)$, and these acceptable aspiration levels, $b_{i}(i=1, \ldots, k)$. Also, $d_{i}^{+}$and $d_{i}^{-}$are, respectively, over- and under-achievement of the $i^{\text {th }}$ goal.

\section{Fuzzy TOPSIS method}

In this section, we review the extended TOPSIS method in fuzzy environment proposed by Jahanshahloo et al. (2006a).The approach to extend the TOPSIS method to the fuzzy data is as following steps:

- First step is, identification the evaluation criteria.

- Step 2 is, generating alternatives.

- Step 3 is, evaluating alternatives in terms of criteria.

- Step 4. Construct the fuzzy decision matrix.

- Step 5 is, identifying the weight of criteria.

- Step 6. Calculate the normalized fuzzy decision matrix as follows:

First, for each fuzzy number $\tilde{x}_{i j}$, we calculate the set of $\alpha$-cut as

$\tilde{x}_{i j}=\left[\left[\tilde{x}_{i j}\right]_{\alpha}^{L},\left[\tilde{x}_{i j}\right]_{\alpha}^{U}\right], \quad \alpha \in[0,1]$

Therefore, each fuzzy number $\tilde{x}_{i j}$ is transform to an interval, now by an approach proposed in Jahanshahloo et al. $(2006, b)$ we can transform this interval in to normalized interval as follows:

$\left[\tilde{n}_{i j}\right]_{\alpha}^{L}=\left[\tilde{x}_{i j}\right]_{\alpha}^{L} / \sqrt{\sum_{i=1}^{m}\left(\left(\left[\tilde{x}_{i j}\right]_{\alpha}^{L}\right)^{2}+\left(\left[\tilde{x}_{i j}\right]_{\alpha}^{U}\right)^{2}\right)}, \quad i=1, \ldots m ; j=1, \ldots, n$,
$\left[\tilde{n}_{i j}\right]_{\alpha}^{U}=\left[\tilde{x}_{i j}\right]_{\alpha}^{U} / \sqrt{\sum_{i=1}^{m}\left(\left(\left[\tilde{x}_{i j}\right]_{\alpha}^{L}\right)^{2}+\left(\left[\tilde{x}_{i j}\right]_{\alpha}^{U}\right)^{2}\right)}, \quad i=1, \ldots m ; j=1, \ldots, n$,

now, interval $\left[\left[\tilde{n}_{i j}\right]_{\alpha}^{L},\left[\tilde{n}_{i j}\right]_{\alpha}^{U}\right]$ is normalized of interval $\left[\left[\tilde{x}_{i j}\right]_{\alpha}^{L},\left[\tilde{x}_{i j}\right]_{\alpha}^{U}\right]$. We can transform this

normalized interval in to a triangular fuzzy number such as $\tilde{N}_{i j}=\left(a_{i j}, b_{i j}, c_{i j}\right)$ such that, $b_{i j}$ is obtained when $\alpha=1$, also by setting $\alpha=0$ we have $a_{i j}=\left[\tilde{n}_{i j}\right]_{\alpha=0}^{L}$ and $c_{i j}=\left[\tilde{n}_{i j}\right]_{\alpha=0}^{U}$ and therefore $\tilde{N}_{i j}$ is a normalized positive triangular fuzzy number.

- Step 7. construct the weighted normalized fuzzy decision matrix

By considering the different importance of each criterion, we can construct the weighted normalized

fuzzy decision matrix as:

$\tilde{v}_{i j}=\tilde{N}_{i j} \cdot \tilde{w}_{j}$

where $\tilde{w}_{j}$ is the weight of $j$ th attribute or criterion.

- Step 8. Identify the fuzzy positive ideal and fuzzy negative ideal solutions

Now, each $\tilde{v}_{i j}$ is normalized fuzzy numbers and their ranges is belong to [0,1]. So, we can identify

The fuzzy positive ideal solution and fuzzy negative ideal solution as:

$\tilde{A}^{+}=\left(\tilde{v}_{1}^{+}, \ldots, \tilde{v}_{n}^{+}\right)$,

$\tilde{A}^{-}=\left(\tilde{v}_{1}^{-}, \ldots, \tilde{v}_{n}^{-}\right)$,

where $\tilde{v}_{j}^{+}=(1,1,1)$ and $\tilde{v}_{j}^{-}=(0,0,0), j=1, \ldots, n$, for each criteria.

- Step 9. Calculate the separation of each alternative 
The separation of each alternative from the fuzzy positive ideal solution, using the distance measurement between two fuzzy number (see Definition 7) can be currently calculated as:

$\tilde{d}_{i}^{+}=\sum_{j=1}^{n} d\left(\tilde{v}_{i j}, \tilde{v}_{j}^{+}\right), \quad i=1, \ldots, m$,

Similarly, the separation from the fuzzy negative ideal solution can be calculated as:

$\tilde{d}_{i}^{-}=\sum_{j=1}^{n} d\left(\tilde{v}_{i j}, \tilde{v}_{j}^{-}\right), \quad i=1, \ldots, m$,

- Step 10. Calculate the closeness coefficient.

A closeness coefficient is defined to determine the ranking order of all alternatives once the $\tilde{d}_{i}^{+}$and $\tilde{d}_{i}^{-}$of each alternative $A_{i}$ has been calculated. The relative closeness of the alternative $A_{i}$ with respect to $\tilde{A}^{+}$and $\tilde{A}^{-}$isdefined as:

$\tilde{R}_{i}=\tilde{d}_{i}^{-} /\left(\tilde{d}_{i}^{-}+\tilde{d}_{i}^{+}\right), \quad i=1, \ldots, m$,

Obviously, an alternative $A_{i}$ is closer to the $\tilde{A}^{+}$and farther from $\tilde{A}^{-}$as $\tilde{R}_{i}$ approaches to 1 . Therefore, according to the closeness coefficient, we can determine the ranking order of all alternatives and select the best one from among a set of feasible alternatives.

\section{Deriving the fuzzy weights of criteria}

In the conventional case, if a pair-wise comparison matrix $A$ be reciprocal and consistent then the weights of each criterion is simply calculated as

$w_{i}=\frac{a_{i j}}{\sum_{k=1}^{n} a_{k j}}, \quad i=1, \ldots, n$.

where $j \in\{1, \ldots, n\}$ is the index of an arbitrary column. That is $a_{i j}=\frac{w_{i}}{w_{j}}$ or equivalently $a_{i j} w_{j}-w_{i}=0$. In the case of inconsistent matrix, the relation (12) is no longer holds. In the case of fuzzy matrix, we must obtain the fuzzy importance weights $\tilde{w}_{i}=\left(w_{i}^{L}, w_{i}^{M}, w_{i}^{U}\right), \quad i=1, \ldots, n$, such that $a_{i j}^{L}=\frac{w_{i}^{L}}{w_{j}^{L}}, a_{i j}^{M}=\frac{w_{i}^{M}}{w_{j}^{M}}$ and $a_{i j}^{U}=\frac{w_{i}^{U}}{w_{j}^{U}}$. From relation () this is equivalent to find $\tilde{w}_{i}=\left(w_{i}^{L}, w_{i}^{M}, w_{i}^{U}\right), i=1, \ldots, n$, such that $a_{i j}^{L} w_{j}^{L}-w_{i}^{L}=0, a_{i j}^{M} w_{j}^{M}-w_{i}^{M}=0$ and $a_{i j}^{U} w_{j}^{U}-w_{i}^{U}=0$.

Therefore in the case of uncertainty, for deriving the fuzzy weights of criteria from inconsistent fuzzy comparison matrix for $i, j=1, \ldots, n$ we introduce deviation variables $p_{i j}^{L}, q_{i j}^{L}, p_{i j}^{M}, q_{i j}^{M}$ and $p_{i j}^{U}, q_{i j}^{U}$, where deviation variables are nonnegative real numbers, but can't be positive at the same time, i.e. $p_{i j}^{L} q_{i j}^{L}=0, p_{i j}^{M} q_{i j}^{M}=0$ and $p_{i j}^{U} q_{i j}^{U}=0$. Now we apply the goal programming method. It is desirable that the deviation variables are kept to be small as possible, which leads to the goal programming model (13).

By solving model (13) the optimal fuzzy weight vector $\tilde{w}_{i}=\left(w_{i}^{L}, w_{i}^{M}, w_{i}^{U}\right), i=1, \ldots, n$, which show the fuzzy importance of each criterion will be obtained. We can use these weights in the process of solving a multiple criteria decision making problem. Also, these weights show that which criterion is more important than others. 
$d^{*}=\min \sum_{i=1}^{n} \sum_{j=1}^{n}\left(p_{i j}^{L}+q_{i j}^{L}+p_{i j}^{M}+q_{i j}^{M}+p_{i j}^{U}+q_{i j}^{U}\right)$

subject to

$$
\begin{array}{lc}
a_{i j}^{L} w_{j}^{L}-w_{i}^{L}+p_{i j}^{L}-q_{i j}^{L}=0, & i, j=1, \ldots, n \\
a_{i j}^{M} w_{j}^{M}-w_{i}^{M}+p_{i j}^{M}-q_{i j}^{M}=0, & i, j=1, \ldots, n \\
a_{i j}^{U} w_{j}^{U}-w_{i}^{U}+p_{i j}^{U}-q_{i j}^{U}=0, & i, j=1, \ldots, n \\
\sum_{j=1}^{n}\left(w_{j}^{L}+w_{j}^{M}+w_{j}^{U}\right)=1, & \\
w_{j}^{M}-w_{j}^{L} \geq 0, & j=1, \ldots, n \\
w_{j}^{U}-w_{j}^{M} \geq 0, & j=1, \ldots, n \\
w_{j}^{L}, p_{i j}^{L}, q_{i j}^{L}, p_{i j}^{M}, q_{i j}^{M}, p_{i j}^{U}, q_{i j}^{U} \geq 0, & i, j=1, \ldots, n
\end{array}
$$

Remark 1. This method is also useable, even if all data of comparison matrix be in exact form. In such case we obtain the crisp weights for criteria.

Theorem 1.The model (13) is always feasible.

\section{Proof.}

Consider the vector $\hat{W}=\left(\hat{w}_{1}, \ldots, \hat{w}_{n}\right)$, where $\tilde{w}_{j}=\left(w_{j}^{L}, w_{j}^{M}, w_{j}^{U}\right)$ is such that

$$
\left\{\begin{array}{lr}
\sum_{j=1}^{3}\left(w_{j}^{L}+w_{j}^{M}+w_{j}^{U}\right)=1, & \\
w_{j}^{M}-w_{j}^{L} \geq 0, & j=1,2,3, \\
w_{j}^{U}-w_{j}^{M} \geq 0, & j=1,2,3, \\
w_{j}^{L} \geq 0, & j=1,2,3 .
\end{array}\right.
$$

Then we define

$$
\left\{\begin{array}{l}
p_{i j}^{L}=\max \left\{-\left(a_{i j}^{L} w_{j}^{L}-w_{i}^{L}\right), 0\right\}, \\
q_{i j}^{L}=\max \left\{\left(a_{i j}^{L} w_{j}^{L}-w_{i}^{L}\right), 0\right\}, \\
p_{i j}^{M}=\max \left\{-\left(a_{i j}^{M} w_{j}^{M}-w_{i}^{M}\right), 0\right\}, \\
q_{i j}^{M}=\max \left\{\left(a_{i j}^{M} w_{j}^{M}-w_{i}^{M}\right), 0\right\}, \\
p_{i j}^{U}=\max \left\{-\left(a_{i j}^{U} w_{j}^{U}-w_{i}^{U}\right), 0\right\}, \\
q_{i j}^{U}=\max \left\{\left(a_{i j}^{U} w_{j}^{U}-w_{i}^{U}\right), 0\right\},
\end{array}\right.
$$

It is clear that $\left(\tilde{W}, p_{i j}^{L}, q_{i j}^{L}, p_{i j}^{M}, q_{i j}^{M}, p_{i j}^{U}, q_{i j}^{U}\right)$ is a feasible solution for model (13).

Remark 2. In order to ranking of these criteria, we assign the rank 1 to the criterion with the maximal value of $\mathrm{M}\left(w_{i}\right)$, etc., in a decreasing order of $\mathrm{M}\left(w_{i}\right)$.

Special Case: The case of matrix with crisp elements.

In the case of matrix with crisp data, in order to deriving the weights of criteria from the inconsistent pair-wise comparison matrix, the goal programming model (13) can be converted to the following model: 
$d^{*}=\min \sum_{i=1}^{n} \sum_{j=1}^{n}\left(p_{i j}+q_{i j}\right)$

subject to

$$
\begin{array}{ll}
a_{i j} w_{j}-w_{i}+p_{i j}-q_{i j}=0, & i, j=1, \ldots, n \\
\sum_{j=1}^{n} w_{j}=1, & \\
w_{j}, p_{i j}, q_{i j} \geq 0, & i, j=1, \ldots, n
\end{array}
$$

where $p_{i j}$ and $q_{i j}$ are deviation variables. By solving model (14) the optimal weight vector $w_{j}, j=1, \ldots, n$, which show the importance of each criterion will be obtained.

Theorem 2.In the case of crisp data, the pairwise comparison matrix A is consistent if and only if $d^{*}=0$.

\section{Proof.}

Let us first prove that, if $d^{*}=0$ then matrix A is consistent.

Since $d^{*}=0$ we have $p_{i j}=q_{i j}=0$. Therefore $a_{i j} w_{j}-w_{i}=0$ and hence $a_{i j}=\frac{w_{i}}{w_{j}}$. This gives $a_{i j} a_{j k}=a_{i k}$, and we conclude that matrix A is consistent.

Conversely, suppose that matrix $\mathrm{A}$ is consistent. That is $a_{i j} a_{j k}=a_{i k}, \quad i, j, k=1, \ldots, n$

Now, if we define

$$
\left\{\begin{array}{l}
\bar{w}_{j}=\frac{a_{j k}}{\sum_{t=1}^{n} a_{t k}}, j=1, \ldots, n \\
\bar{p}_{i j}=\bar{q}_{i j}=0
\end{array}\right.
$$

then it is easy to check that $\left(\bar{W}, \bar{p}_{i j}, \bar{q}_{i j}\right)$ is feasible for model (14). Since model (14) has minimization form, we conclude that $d^{*}=0$.

Theorem 3. Model (14) is always feasible.

\section{Proof.}

By Theorem 1, proof is evident. $\square$

\section{Illustrating examples}

In this section we present some illustrating example showing that the proposed approach is a convenient tool not only for calculating the fuzzy weights of criteria from a pair-wise comparison matrices with fuzzy elements, but also for calculating the weights of criteria of crisp pair-wise comparison matrices.

\subsection{Example 1: Matrix with crisp elements}

Consider $3 \times 3$ reciprocal matrix A with crisp elements $A=\left[\begin{array}{ccc}1 & \frac{1}{2} & \frac{1}{4} \\ 2 & 1 & \frac{1}{4} \\ 4 & 4 & 1\end{array}\right]$ 
We can easily check that the pairwise comparison matrix A is reciprocal but it is inconsistent. Now, for deriving the weights of criteria we apply a goal programming model (14) to matrix A. Therefore we must solve the following goal programming model

$$
\begin{aligned}
d^{*}=\min & p_{12}+q_{12}+p_{13}+q_{13}+p_{21}+q_{21} \\
& +p_{23}+q_{23}+p_{31}+q_{31}+p_{32}+q_{32}
\end{aligned}
$$

subject to

$$
\begin{aligned}
& 0.50 w_{2}-w_{1}+p_{12}-q_{12}=0, \\
& 0.25 w_{3}-w_{1}+p_{13}-q_{13}=0, \\
& 2.00 w_{1}-w_{2}+p_{21}-q_{21}=0, \\
& 0.25 w_{3}-w_{2}+p_{23}-q_{23}=0, \\
& 4.00 w_{1}-w_{3}+p_{31}-q_{31}=0, \\
& 4.00 w_{2}-w_{3}+p_{32}-q_{32}=0, \\
& w_{1}+w_{2}+w_{3}=1, \quad 1 \leq i, j \leq 3 . \\
& w_{i}, p_{i j}, q_{i j} \geq 0, \quad
\end{aligned}
$$

By solving model (15), we obtain the optimal vector $W=\left(w_{1}, w_{2}, w_{3}\right)$. We assign the rank 1 to the criteria with the maximal value of $w_{j}$, etc., in a decreasing order of $w_{j}$. The result is shown in Table 1. The optimal objective of model (15) is $d^{*}=0.249$, which shows that the pairwise comparison matrix A is inconsistent by Theorem 2 .

\section{Table 1}

The result of proposed method for example 1 .

\begin{tabular}{lcc}
\hline Criteria & The obtained weights & Rank of criteria \\
\hline 1 & $w_{1}=0.16667$ & 2 \\
2 & $w_{2}=0.16667$ & 2 \\
3 & $w_{3}=0.66666$ & 1 \\
\hline
\end{tabular}

In this example the rank order of these criteria is as

$$
w_{3}>w_{1} \sim w_{2}
$$

The results of ranking these criteria are shown in last column of Table 1.

\subsection{Example 2: Matrix with fuzzy elements}

Consider the following $3 \times 3$ reciprocal matrix $\tilde{A}$ with triangular fuzzy elements:

$$
\tilde{A}=\left[\begin{array}{ccc}
(1,1,1) & (2,3,4) & (4,5,6) \\
\left(\frac{1}{4}, \frac{1}{3}, \frac{1}{2}\right) & (1,1,1) & (3,4,5) \\
\left(\frac{1}{6}, \frac{1}{5}, \frac{1}{4}\right) & \left(\frac{1}{5}, \frac{1}{4}, \frac{1}{3}\right) & (1,1,1)
\end{array}\right]
$$

For deriving the fuzzy weights of criteria, similar to model (15), we construct the goal programming model (16) as 


$$
\begin{array}{cl}
\min \sum_{i=1}^{3} \sum_{j=1}^{3}\left(p_{i j}^{L}+q_{i j}^{L}+p_{i j}^{M}+q_{i j}^{M}+p_{i j}^{U}+q_{i j}^{U}\right) & \\
\text { subject to } & 0.2000 w_{1}^{M}-w_{3}^{M}+p_{31}^{M}-q_{31}^{M}=0, \\
2.0000 w_{2}^{L}-w_{1}^{L}+p_{12}^{L}-q_{12}^{L}=0, & 0.2500 w_{2}^{M}-w_{3}^{M}+p_{32}^{M}-q_{32}^{M}=0, \\
4.0000 w_{3}^{L}-w_{1}^{L}+p_{13}^{L}-q_{13}^{L}=0, & 4.0000 w_{2}^{U}-w_{1}^{U}+p_{12}^{U}-q_{12}^{U}=0, \\
0.2500 w_{1}^{L}-w_{2}^{L}+p_{21}^{L}-q_{21}^{L}=0, & 6.0000 w_{3}^{U}-w_{1}^{U}+p_{13}^{U}-q_{13}^{U}=0, \\
3.0000 w_{3}^{L}-w_{2}^{L}+p_{23}^{L}-q_{23}^{L}=0, & 0.5000 w_{1}^{U}-w_{2}^{U}+p_{21}^{U}-p_{21}^{U}=0, \\
0.1667 w_{1}^{L}-w_{3}^{L}+p_{31}^{L}-q_{31}^{L}=0, & 5.0000 w_{3}^{U}-w_{2}^{U}+p_{23}^{U}-q_{23}^{U}=0, \\
0.2000 w_{2}^{L}-w_{3}^{L}+p_{32}^{L}-q_{32}^{L}=0, & 0.2500 w_{1}^{U}-w_{3}^{U}+p_{31}^{U}-q_{31}^{U}=0, \\
3.0000 w_{2}^{M}-w_{1}^{M}+p_{12}^{M}-q_{12}^{M}=0, & 0.3333 w_{2}^{U}-w_{3}^{U}+p_{32}^{U}-q_{32}^{U}=0, \\
5.0000 w_{3}^{M}-w_{1}^{M}+p_{13}^{M}-q_{13}^{M}=0, & \sum_{j=1}^{3}\left(w_{j}^{L}+w_{j}^{M}+w_{j}^{U}\right)=1, \\
0.3333 w_{1}^{M}-w_{2}^{M}+p_{21}^{M}-q_{21}^{M}=0, & w_{j}^{L} \leq w_{j}^{M} \leq w_{j}^{U}, j=1,2,3, \\
4.0000 w_{3}^{M}-w_{2}^{M}+p_{23}^{M}-q_{23}^{M}=0, & w_{j}^{L}, p_{i j}^{L}, q_{i j}^{L}, p_{i j}^{M}, q_{i j}^{M}, p_{i j}^{U}, q_{i j}^{U} \geq 0,1 \leq i, j \leq 3 . \\
0.2000 w_{1}^{M}-w_{3}^{M}+p_{31}^{M}-q_{31}^{M}=0, &
\end{array}
$$

The results of the model (16) are shown in Table 2.

Table 2

The fuzzy weights for example 2

\begin{tabular}{llcc}
\hline Criteria & The obtained fuzzy weights & $M\left(\tilde{w}_{j}\right)$ & Rank of Criteria \\
\hline 1 & $\tilde{w}_{1}=(0.1471,0.2206,0.2941)$ & 0.2206 & 1 \\
2 & $\tilde{w}_{2}=(0.0735,0.0735,0.0735)$ & 0.0735 & 2 \\
3 & $\tilde{w}_{3}=(0.0245,0.0441,0.0490)$ & 0.040425 & 3 \\
\hline
\end{tabular}

It can be seen that in the above example we derive the fuzzy weights of criteria when the elements of its pair-wise comparison matrix are in the form of triangular fuzzy numbers. In order to compare and rank these criteria, we use the ranking function $\mathrm{M}($.$) . Results are shown in the two last columns of$ Table 2.

\subsection{A case study}

Suppose that a software company desires to hire a system analysis engineer. After preliminary screening, three candidates $A_{1}, A_{2}$ and $A_{3}$ remain for further evaluation. The company asks a decision-maker, to conduct the interview and to select the most suitable candidate. Tree benefit criteria are considered:

1) personality $\left(C_{1}\right)$,

2) past experience $\left(C_{2}\right)$,

3) self-confidence $\left(C_{3}\right)$.

The hierarchical structure of this decision problem is shown as Fig. 2.

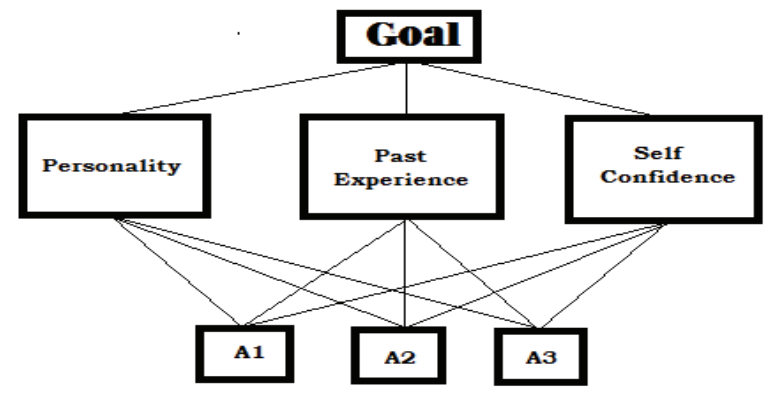

Fig. 2. The hierarchical Structure

The decision maker provides a fuzzy pair-wise comparison matrix between criteria as Table 3 . 
Table 3

The fuzzy pair-wise comparison matrix

\begin{tabular}{cccc}
\hline & $C_{1}$ & $C_{2}$ & $C_{3}$ \\
\hline $\boldsymbol{C}_{\mathbf{1}}$ & $(1,1,1)$ & $(0.1,0.125,0.2)$ & $(0.5,0.75,1)$ \\
$\boldsymbol{C}_{\mathbf{2}}$ & $(5,8,10)$ & $(1,1,1)$ & $(1,2,5)$ \\
$\boldsymbol{C}_{\mathbf{3}}$ & $(1,1.333,2)$ & $(0.2,0.5,1)$ & $(1,1,1)$ \\
\hline
\end{tabular}

The decision-maker uses the triangular fuzzy rating variables to evaluate the rating of alternatives with respect to each criterion and present it in Table 4.

\section{Table 4}

The fuzzy ratings of the three candidates by decision maker under all criteria

\begin{tabular}{lccc}
\hline & $C_{1}$ & $C_{2}$ & $C_{3}$ \\
\hline $\boldsymbol{A}_{\mathbf{1}}$ & $(5,7,9)$ & $(4,7,10)$ & $(5,6,7)$ \\
$\boldsymbol{A}_{\mathbf{2}}$ & $(3,4,6)$ & $(9,9,10)$ & $(7,8,9)$ \\
$\boldsymbol{A}_{\mathbf{3}}$ & $(7,9,10)$ & $(3,3,5)$ & $(3,4,7)$ \\
\hline
\end{tabular}

The proposed method is currently applied to solve this problem and the computational procedure is summarized as follows:

- Until now, four steps of algorithm have been done.

- Step 5: Determine the importance of the criteria.

By using the information of Table 3 and the proposed goal programming method the fuzzy weights of criteria are obtained as Table 5.

Table 5

The fuzzy weights for criteria

\begin{tabular}{lccc}
\hline Criteria & $C_{1}$ & $C_{2}$ & $C_{3}$ \\
\hline Fuzzy weight & $(0.0256,0.0513)$ & $(0,0.2051,0.5128)$ & $(0,0.1026,0.1026)$ \\
\hline
\end{tabular}

- Step 6. Calculate the normalized fuzzy decision matrix.

- Step 7. Calculate the weighted normalized fuzzy decision matrix.

Normalized fuzzy decision matrix and weighted normalized fuzzy decision matrix are given in Tables 6 and 7 , respectively.

Table 6

The normalized fuzzy decision matrix

\begin{tabular}{cccc}
\hline & $C_{1}$ & $C_{2}$ & $C_{3}$ \\
\hline $\boldsymbol{A}_{\mathbf{1}}$ & $(0.289,0.410,0.520)$ & $(0.220,0.420,0.550)$ & $(0.309,0.368,0.432)$ \\
$\boldsymbol{A}_{\mathbf{2}}$ & $(0.173,0.234,0.346)$ & $(0.495,0.540,0.550)$ & $(0.432,0.490,0.556)$ \\
$\boldsymbol{A}_{\mathbf{3}}$ & $(0.404,0.527,0.577)$ & $(0.165,0.180,0.275)$ & $(0.185,0.245,0.432)$ \\
\hline
\end{tabular}

Table 7

The weighted normalized fuzzy decision matrix

\begin{tabular}{cccc}
\hline & $C_{1}$ & $C_{2}$ & $C_{3}$ \\
\hline $\boldsymbol{A}_{\mathbf{1}}$ & $(0,0.0105,0.0267)$ & $(0,0.0861,0.2820)$ & $(0,0.0378,0.0443)$ \\
$\boldsymbol{A}_{\mathbf{2}}$ & $(0,0.0060,0.0178)$ & $(0,0.1108,0.2820)$ & $(0,0.0503,0.0570)$ \\
$\boldsymbol{A}_{\mathbf{3}}$ & $(0,0.0135,0.0296)$ & $(0,0.0369,0.1410)$ & $(0,0.0251,0.0443)$ \\
\hline
\end{tabular}

- Step 8. Identify the fuzzy positive ideal and fuzzy negative ideal solutions.

- Step 9. Calculate the separation of each alternative. 
The separation measures are shown in Table 8 .

Table 8

The closeness coefficient and ranking of criteria

\begin{tabular}{ccccc}
\hline & $\tilde{d}_{i}^{+}$ & $\tilde{d}_{i}^{-}$ & $\tilde{R}_{i}$ & Rank \\
\hline $\boldsymbol{A}_{\mathbf{1}}$ & 2.845681 & 0.220428 & 0.071892 & 2 \\
$\boldsymbol{A}_{\mathbf{2}}$ & 2.833456 & 0.229657 & 0.074975 & 1 \\
$\boldsymbol{A}_{\mathbf{3}}$ & 2.905306 & 0.132362 & 0.043573 & 3 \\
\hline
\end{tabular}

- Step 10. Calculate the closeness coefficient.

The closeness coefficients, which are defined to determine the ranking order of all alternatives by calculating the distance to both the "fuzzy positive-ideal solution" and the "fuzzy negative-ideal solution" simultaneously, are given in fourth column of Table 8. Now a preference order can be ranked according to the order of $\tilde{R}_{i}$. Therefore, the best alternative is the one with the shortest distance to the fuzzy positive ideal solution and with the longest distance to the fuzzy negative ideal solution. According to the closeness coefficient, ranking the preference order of these alternatives is as Table 8 .

\section{Conclusion}

Finding the weights of criteria has been one of the most important issues in the field of decision making. In this paper, we have investigated the problem of deriving the fuzzy weights of criteria from the pair-wise comparison matrix with fuzzy elements. In the presented method the goal programming method have been put forward. We drive the fuzzy weights of criteria by using the goal programming method. The proposed method is applied for two pair-wise comparison matrices with crisp and fuzzy elements, respectively. In addition, we applied the proposed method to solve multi-criteria decision problem based on TOPSIS method. In order to compare the criteria we use the ranking function proposed by Asady and Zendehnam. The approach is illustrated by using some examples. In addition, the proposed method can be applied to solve other multi-criteria decision problems.

\section{References}

Abo-Sinna, M.A., \& Amer, A.H. (2005). Extensions of TOPSIS for multi-objective large-scale nonlinear programming problems. Applied Mathematics and Computation, 162, 243-256.

Asady, B., \& Zendehnam, M. (2007). Ranking of fuzzy numbers by minimize distance, Applied Mathematical Modeling,31, 2589-2598.

Bellman, R.E., \& Zadeh, L.A. (1970). Decision making in a fuzzy environment. Management Science, 17, 141-164.

Barzilai, J. (1997). Deriving weights from pairwise comparison matrices. Journal of Operational Research Society, 48, 1226-1232.

Charnes, A., \& Cooper, W.W. (1961). Management Model and Industrial Application of Linear Programming, $1^{\text {st }}$ ed., Wiley, New York.

Chen, C.T. (2000). Extensions of the TOPSIS for group decision-making under fuzzy environment. Fuzzy Sets and Systems, 114, 1-9.

Chen, S. J., \& Hwang C. L. (1992). Fuzzy Multiple Attribute Decision Making: Methods and Applications, Springer-Verlag. Berlin.

Cogger, K.O., \& Yu, P.L. (1985). Eigen weight vectors and least distance approximation for revealed preference in pairwise weight ratios. Journal of Optimization Theory and Applications, 46, 483491. 
Crawford, G., \& Williams, C.A. (1985). A note on the analysis of subjective judgment matrices. Journal of Mathematical Psychology, 29, 387-405.

Geoffrion, A.M., Dyer, J.S., \& Feinberg, A. (1972). An interactive approach for multicriterion optimization with an application to operation of an academic department. Management Science, 19, 357-368.

Haimes, Y.Y. (1980). The surrogate worth trade-off (SWT) method and its extensions, in: G. Fandel and T. Gal (eds.). Multiple Criteria Decision Making Theory and Application, Springer-Verlag, New York.

Hwang C.L., \& Yoon K. (1981). Multiple Attribute Decision Making Methods and Applications. Springer, Berlin Heidelberg.

Islam, R., Biswal, M.P., \& Alam, S.S. (1997). Preference programming and inconsistent interval judgments. European Journal of Operational Research, 97, 53-62.

Izadikhah, M. (2009). Using the Hamming distance to extend TOPSIS in a fuzzy environment. Journal of Computational and Applied Mathematics, 231, 200-207.

Jahanshahloo, G.R., Hosseinzadeh Lotfi F., Izadikhah M. (2006). An algorithmic method to extend TOPSIS for decision-making problems with interval data. Applied Mathematics and Computation, $175,1375-1384$.

Jahanshahloo, G.R., Hosseinzadeh Lotfi, F., \& Izadikhah, M. (2006). Extension of the TOPSIS method for decision-making problems with fuzzy data. Applied Mathematics and Computation, 181(2), 1544-1551.

Ramik, J., \& Korviny, P. (2010). Inconsistency of pair-wise comparison matrix with fuzzy elements based on geometric mean. Fuzzy Sets and Systems, 161, 1604-1613.

Saaty, T.L. (1980). The Analytic Hierarchy Process, McGraw-Hill, New York.

Salo A, \& Haimalainen R. (1997). On the measurement of preferences in the analytic hierarchy process. Journal of Multi-Criteria Decision Analysis, 6, 309-319.

Takeda, E., Cogger, K.O., \& Yu, P.L. (1987). Estimating criterion weights using eigenvectors: A comparative study. European Journal of Operational Research, 29, 360-369.

Wang, Y-M. (2006). On lexicographic goal programming method for generating weights from inconsistent interval comparison matrices. Applied Mathematics and Computation, 173, 985-991.

Zionts, S., \& Wallenius, J. (1976). An interactive programming method for solving the multiple criteria problem. Management Science, 22, 652-663.

Zadeh, L.A. (1965). Fuzzy sets. Information and Control, 8, 338-353. 